\title{
Spoof surface plasmon polaritons based notch filter for ultra-wideband microwave waveguide
}

\author{
Xiao, Binggang; Li, Sheng-Hua ; Xiao, Sanshui
}

Published in:

Optics Communications

Link to article, DOI:

10.1016/j.optcom.2016.04.019

Publication date:

2016

Document Version

Peer reviewed version

Link back to DTU Orbit

Citation (APA):

Xiao, B., Li, S-H., \& Xiao, S. (2016). Spoof surface plasmon polaritons based notch filter for ultra-wideband microwave waveguide. Optics Communications, 374, 13-17. https://doi.org/10.1016/j.optcom.2016.04.019

\section{General rights}

Copyright and moral rights for the publications made accessible in the public portal are retained by the authors and/or other copyright owners and it is a condition of accessing publications that users recognise and abide by the legal requirements associated with these rights.

- Users may download and print one copy of any publication from the public portal for the purpose of private study or research.

- You may not further distribute the material or use it for any profit-making activity or commercial gain

- You may freely distribute the URL identifying the publication in the public portal

If you believe that this document breaches copyright please contact us providing details, and we will remove access to the work immediately and investigate your claim. 


\title{
Spoof Surface Plasmon Polaritons Based Notch Filter for Ultra-wideband Microwave Waveguide
}

\author{
Binggang Xiao $^{\mathrm{a}, \mathrm{b}}$, Sheng Kong ${ }^{\mathrm{a}}$, Sanshui Xiao \\ ${ }^{a}$ College of Information Engineering, China Jiliang University, Hangzhou 310018, China, \\ ${ }^{\mathrm{b}}$ Department of Photonics Engineering, Technical University of Denmark, Lyngby 2800, Denmark
}

\begin{abstract}
Spoof surface plasmon polaritons based notch filter for ultra-wideband microwave waveguide is proposed. Owing to subwavelength confinement, such a filter has advantage in the structure size without sacrificing the performance. The spoof SPP based notch is introduced to suppress the WLAN and satellite communication interference simultaneously. Both the cutoff frequency and the notch frequency are sensitive to the structure parameters, and the cut-off frequency can reach $20 \mathrm{GHz}$. An adiabatic transition relying on gradient hole-size and flaring ground is designed to effectively couple energy into spoof SPP waveguide. The result shows its cut-off frequency of $17.4 \mathrm{GHz}$ with the insertion loss better than $3 \mathrm{~dB}$ during the whole pass-band, while having more than $20 \mathrm{~dB}$ rejections at $5.36 \mathrm{GHz}$ and $9.32 \mathrm{GHz}$ with $10 \mathrm{~dB}$ fractional bandwidth $1.07 \%$ and $0.74 \%$ respectively to avoid the existing WLAN and satellite communication signals. Due to planar structures proposed here, it is easy to integrate in the microwave integrated systems, which can play an important role in the microwave communication circuit and system.
\end{abstract}

Index Terms - Notch band, UWB filter, Spoof Surface plasmon Polaritons.

\section{INTRODUCTION}

$\mathrm{U}$ LTRAWIDEBAND (UWB), the unlicensed use of a

wide bandwidth in 3.1-10.6 GHz frequency band, was authorized by the Federal Communications Commission to transmit information in 2002[1]. The design of UWB devices such as filters, antennas and low noise amplifiers are essential for the dependable use of UWB systems in sensor, radar, and tracking applications with high data transmission [2-3]. Filters are generally required in many types of UWB systems to suppress unwanted harmonics and spurious signals [1-3]. Because of the collocation of the UWB frequency spectrum with the frequency band reserved for some existing wireless networking systems, such as WLAN and satellite communications, there is a desire to eliminate these signals from the 3.1-10.6 GHz range to avoid the possible interference between these systems simultaneously. In order to avoid the undesired WLAN and satellite signals for UWB systems, many structures have been proposed over the years, such as complementary split ring resonators, stepped implement configuration and defected ground structure [1-3].

Surface plasmon polaritons are electromagnetic waves in the optical frequency region, which are bound at a metal-dielectric interface, and they are propagating parallel to the interface and decaying exponentially in the direction vertical to the interface, owing to the negative permittivity behavior of metal [4]. In the far-infrared, terahertz, and microwave frequency bands, metal behaves assemble as perfectly electrical conductors, and SPPs cannot exit at the metal surface[5]. A new concept to introduce SPP at the metal surface was theoretically proposed and experimentally demonstrated [4, 6-9], which confirms that the metal surface also supports highly confined SPP at low frequencies, so-called spoof SPPs. The spoof SPP structures have been proposed to transform the traditional Somerfield or Zen neck surface waves into the highly confined spoof SPPs. Generally, spoof SPP structures are composed by metal surfaces decorated with 1D arrays of sub wavelength grooves, $2 \mathrm{D}$ arrays of sub wavelength holes/dimples, or 3D metal wire in which a periodic array of radical grooves is drill[ $4,10-15]$. To solve the shortcomings in the fabrication and integration for most of the currently existing 3D spoof SPP structures, an ultrathin spoof SPPs one-dimensional grooves structure in the microwave frequencies has been proposed by Cui et al, which can be bent, twisted, and wrapped to arbitrary surfaces to produce conformal surface plasmons (CSPs)[5, 15-22]. And these structures have been used to design broadband planar and flexible SPP waveguide, $90^{\circ}$ bend, beam splitter, and ring resonator in the microwave frequency recently by Cui et al [21, 23-25].

Being inspired by T. J. Cui group's excellent work $[7,10,14,17-18,21,23-24]$ in surface plasmon polaritons, we propose a notch filter for ultra-wideband microwave waveguide using a ultrathin spoof SPPs planar holes structure based ring resonator in this paper. The filter was analyzed theoretically and simulated using CST software. The result shows that it has two sharp notched bands which are located at $5.36 \mathrm{GHz}$ and $9.32 \mathrm{GHz}$ with a $10 \mathrm{~dB}$ notched fractional bandwidth of $1.61 \% / 1.29 \%$ and more than $20 \mathrm{~dB}$ rejection respectively. According to the reference [22], the dispersion curve of rectangle holes might be more deviated from the light line and 
the cut-off frequency might be lower compared to rectangular groove, so rectangle holes were used here instead of grooves. Compared to the conventional structures (e.g. SIR, CSRR, DGS), the proposed Spoof SPPs structure has some significant advantages such as UWB, high-confinement, adjustability and easy integration which are very important for the microwave circuits and systems.

\section{THEORY OF SPOOF SPPS WAVEGUIDE}

The spoof SPPs waveguide that we studied consists of a periodic array of drilled holes in a metal strip placing on top of a substrate with its relative dielectric constant $\left(\varepsilon_{h}\right)$ of 3.3 , loss tangent of 0.003 , and thickness $\left(\mathrm{t}_{1}\right)$ of $0.5 \mathrm{~mm}$. The drilled holes in the metal strip are characterized by the width (a), the length (b), and the periodicity (p) as illustrated in Fig.1 (a), where $h$ is the width of the metal strip. The dispersion property of the spoof SPPs waveguide structure can be numerically calculated from a finite-element method (FEM) based Eigen-frequency solver. Here we employ the commercial software of COMSOL Multi-physics. Of course, the electromagnetic property of the spoof SPPs strongly depends on the geometry parameters. Fig. 1(b) illustrates the dispersions of spoof SPPs as a function of $b$ when $\mathrm{p}=5 \mathrm{~mm}, \mathrm{a}=2 \mathrm{~mm}, \mathrm{~h}=6 \mathrm{~mm}$, and $\mathrm{t}=0.018 \mathrm{~mm}$. For a low frequency, the dispersion curve for the spoof SPPs is quite close to the light line, while the wave vector for the spoof SPPs becomes larger than that in vacuum, indicating that the mode for the spoof SPPs is strongly confined near the structure. The right insert in Fig.1 (b) shows the normal electric field distribution in the $\mathrm{yz}$ plane when $\mathrm{k}=0.8 \pi / \mathrm{p}$ and $\mathrm{b}=4.8 \mathrm{~mm}$, showing a good confinement to guide the EM waves along the $\mathrm{x}$ direction. As $b$ increases, the dispersion curve deviates further from the light line and the cutoff frequency becomes lower as shown in Fig.1 (b). The cut-off frequency for the structure with $\mathrm{b}=2.4 \mathrm{~mm}$ is $25 \mathrm{GHz}$, moving to $17.4 \mathrm{GHz}$ for the case of $\mathrm{b}=4.8 \mathrm{~mm}$. The left insert shows the top view of the unit cell that is used for the calculation of the dispersion. To realize an ultra-wideband microwave waveguide, the influence of the structure parameters (including the parameters for drilled-holes and the width of the metal strip) to the cut-off frequency is systemically studied and shown in Fig.1(c). Seen from Fig.1(c), the cut-off frequency of the spoof SPPs depends on all structure parameters, and particularly it is quite sensitive to the height of drilled holes as indicated by the black line in Fig. 1(c).

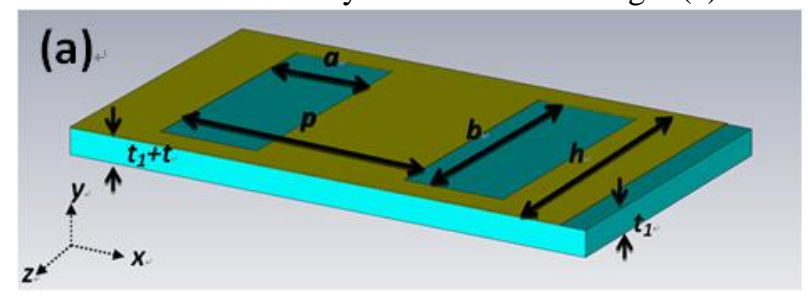

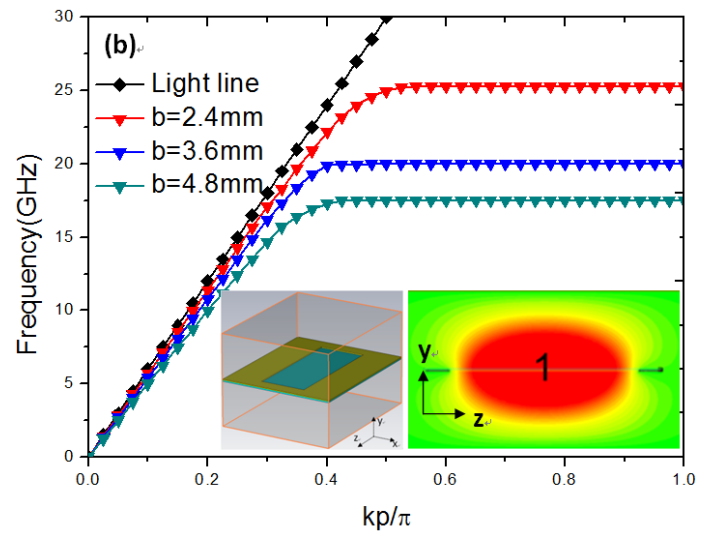

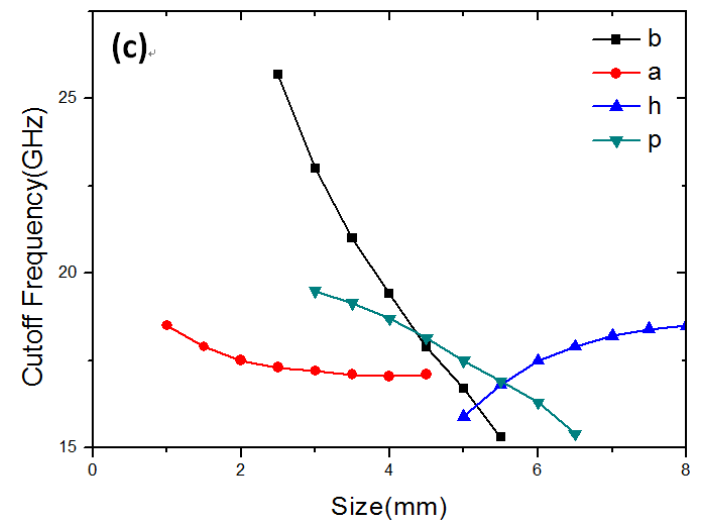

Fig.1.(a) The schematic diagram and geometric parameters of proposed Spoof SPPs structure, in which $\mathrm{a}, \mathrm{b}, \mathrm{p}$, and $\mathrm{h}$ are the width, length, period, and height of the metal hole.(b) Dispersion relations of the spoof SPPs waveguide in microwave regime, where the black line indicates the light line, the red line, blue line and green line represent the spoof SPPs waveguides with $b=2.4 \mathrm{~mm}$, $\mathrm{b}=3.6 \mathrm{~mm}$ and $4.8 \mathrm{~mm}$ respectively. Here, we set $\mathrm{p}=5 \mathrm{~mm}, \mathrm{a}=2 \mathrm{~mm}, \mathrm{~h}=6 \mathrm{~mm}$, $\mathrm{t}=0.018 \mathrm{~mm}$ and $\mathrm{t}_{1}=0.5 \mathrm{~mm}$. The inset shows the normal electric field distribution in the yz plane at $\mathrm{k}=0.8 \pi / \mathrm{p}$. (c) Cut-off frequency versus structure parameters of the length (b), the width (a), the height $(\mathrm{h})$ and the periodicity (p) with $\mathrm{t}=0.018 \mathrm{~mm}$ and $\mathrm{t}_{1}=0.5 \mathrm{~mm}$ when parameters of $\mathrm{a}=2 \mathrm{~mm}, \mathrm{p}=5 \mathrm{~mm}, \mathrm{~h}=6 \mathrm{~mm}$ are fixed (black line), when parameters of $b=4.8 \mathrm{~mm}, \mathrm{p}=5 \mathrm{~mm}, \mathrm{~h}=6 \mathrm{~mm}$ are fixed (red line), when parameters of $b=4.8 \mathrm{~mm}, a=2 \mathrm{~mm}, \mathrm{p}=5 \mathrm{~mm}$ are fixed(blue line) and when parameters of $\mathrm{b}=4.8 \mathrm{~mm}, \mathrm{a}=2 \mathrm{~mm}, \mathrm{~h}=6 \mathrm{~mm}$ are fixed (green line).

\section{PROPOSED NOTCH FILTER WITH TWO NOTCH BANDS}

Relying on the spoof SPPs waveguide mentioned above, a notch filter is proposed, as illustrated in the insert of Fig.2. Here we choose $\mathrm{p}=5 \mathrm{~mm}, \mathrm{a}=2 \mathrm{~mm}, \mathrm{~b}=4.8 \mathrm{~mm}, \mathrm{~h}=6 \mathrm{~mm}$, and $\mathrm{t}=0.018 \mathrm{~mm}$, where its cut-off frequency is around $17.4 \mathrm{GHz}$. In order to avoid the undesired WLAN and satellite signal (working at $5.36 \mathrm{GHz}$ and $9.32 \mathrm{GHz}$ ) for UWB systems, a ring resonator based on the drilled holes metal strip is introduced here with its parameters for the holes same as the metal strips above. The mechanism here is that when a part of the input energy is coupled into the ring and then coupled to the waveguide again after a circulation, the two beams in the right part of waveguide interfere with each other, resulting in the modulation of the output signals. The phase change around the ring can be approximately evaluated by $\Delta \varnothing=\frac{4 \pi^{2} n_{\text {eff }}(\lambda) R}{\lambda}$ , in which $\lambda$ is the wavelength in the free space and $n_{\text {eff }}$ is the 
effective mode index[6,14]. When $\Delta \varnothing=(2 m+1) \pi$, the ring resonator works at the closed state, indicating that the input signal will be cut off $[4,10,14]$, otherwise the signal can be transmitted to the output. As depicted in Fig.2 (a), the central frequency of the notch band largely depends on $\mathrm{R}$ which is consistent with the above theoretical analysis. When the outside radius $\mathrm{R}$ of the ring resonator increases, the notch frequency will decrease a lot. With the decrease of the gap size d, the coupling strength of the proposed filter increases, leading to redshift of the resonance.

In order to simultaneously suppress the undesired WLAN and satellite signals working at the central frequency $5.36 \mathrm{GHz}$ and $9.32 \mathrm{GHz}$ for the ultra-wideband microwave waveguide, by careful optimizing the parameters of the ring resonator, two sharp notched bands appearing exactly at $5.36 \mathrm{GHz}$ and 9.32 $\mathrm{GHz}$ are realized when $\mathrm{R}=9 \mathrm{~mm}$ and $\mathrm{d}=0.1 \mathrm{~mm}$. No higher harmonics is observed here, because the high resonant frequency has been greater than the cutoff frequency of the waveguide. The ring's outer radius here is about half wavelength at the cut-off frequency $(17.4 \mathrm{GHz})$. The $10 \mathrm{~dB}$ fractional bandwidth of two notched bands here are $1.07 \%$ and $0.74 \%$ respectively with rejection greater than $25 \mathrm{~dB}$, as shown in Fig.3. In the whole passing band, the insertion loss is lower than $1 \mathrm{~dB}$. The simulated electric fields $\left(\mathrm{E}_{\mathrm{y}}\right)$ at $5.36 \mathrm{GHz}, 8 \mathrm{GHz}$ and $9.32 \mathrm{GHz}$ are shown in the insert of Fig.3, which clearly demonstrates on-off transmission and two different orders of the resonant states. The first order of the resonance is around $5.36 \mathrm{GHz}$ providing the first notching band and the second order of the resonant frequency is $9.32 \mathrm{GHz}$ resulting in the second notching band. As a comparison, the simulated electric field at $8 \mathrm{GHz}$ is offered to illustrate the passing band case, indicating that SPP waves can travel around the ring smoothly and SPP waveguide.

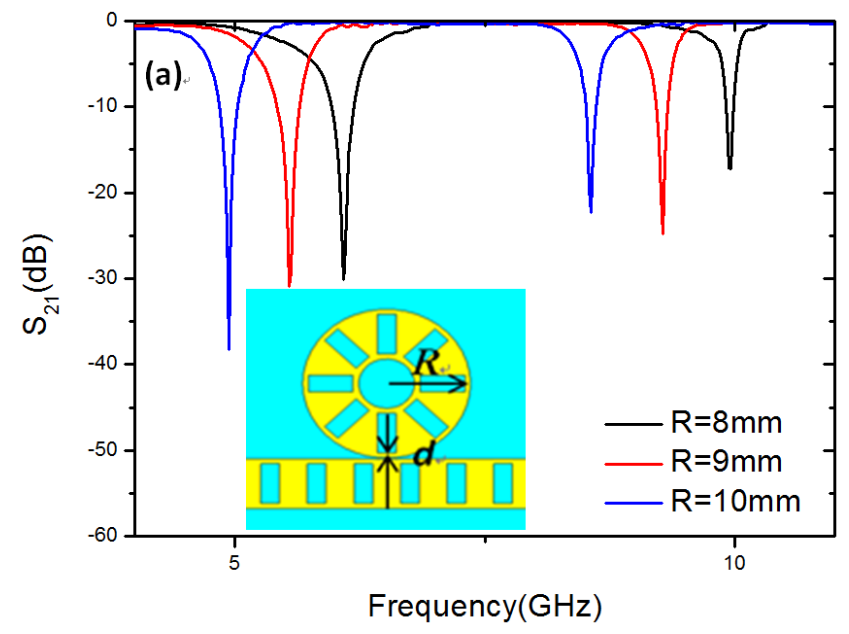

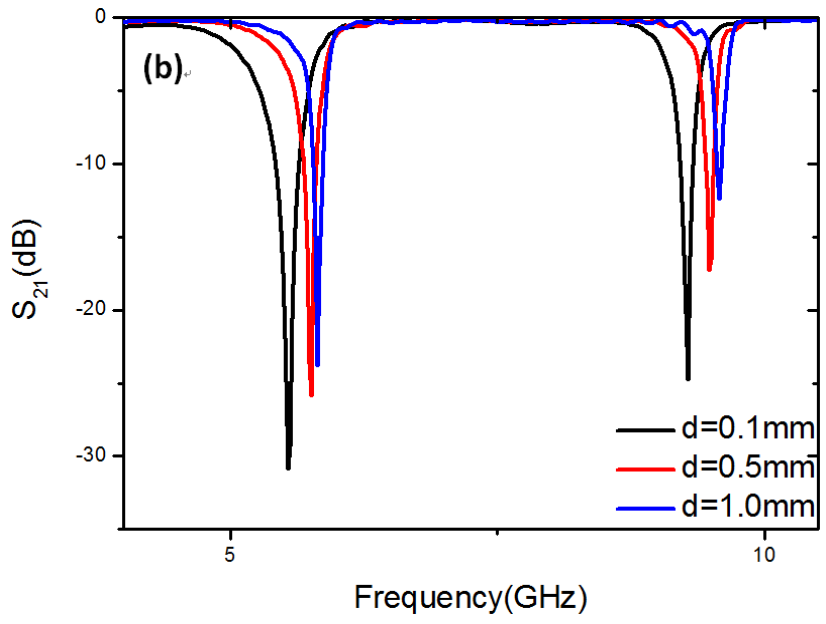

Fig.2.(a) The influence on the $S_{21}$-parameter magnitude of the structure with the change of ring's outside radius. The insert of Fig.2(a) indicated the geometry parameters of the proposed notch filter in which $\mathrm{d}$ is the gap between the ring resonator and spoof SPPs waveguide and $\mathrm{R}$ is the outside radius of the ring resonator. (b) The influence on the $S_{21}$-parameter magnitude of the structure with the change of the gap d between ring and straight waveguide.

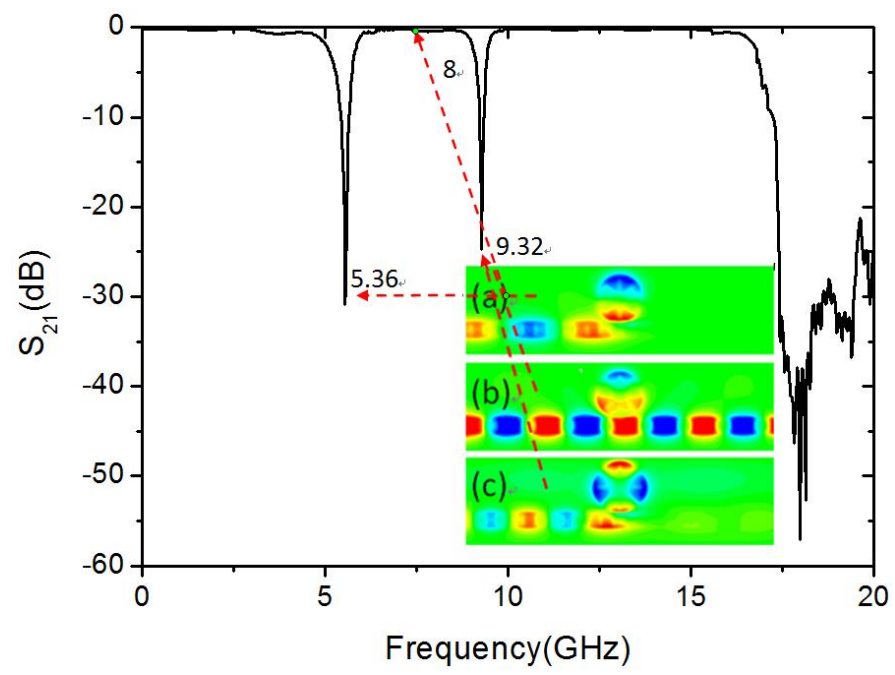

Fig.3.The simulated S-parameter of the UWB LPF in the microwave frequency. The inset shows the simulated electric field (Ey) of UWB LPF at (a) $5.36 \mathrm{GHz}$, (b) $8 \mathrm{GHz}$, (c) $9.32 \mathrm{GHz}$.

\section{HIGH EFFICIENCY CONVERSION FOR THE PROPOSED FILTER}

It is known that it remains challenging to feed/extract energy into/from the spoof SPPs metal strips. In the microwave frequency, conventional coplanar waveguide (CPW) lines are commonly used in integrated circuits. The electric-field vectors in the CPW are in the same plane as those of spoof SPPs [4, 5, 10]. In order to achieve high-efficiency conversion from the CPW line to the spoof SPP waveguide, an adiabatic transition relying on gradient hole-size and flaring ground is designed to overcome the impedance mismatch between CPW and the spoof SPPs. The whole system was schematically shown in Fig.4. The first part is a CPW, which supports quasi-transverse electric and magnetic mode of conventional guided waves. The dimension of CPW is designed as $\mathrm{h}=3 \mathrm{~mm}, \mathrm{w}=15 \mathrm{~mm}$, $\mathrm{g}=0.5 \mathrm{~mm}$, with having $50 \mathrm{ohm}$ impendence. The second part is 
the transition part to make an effective transition from the conventional CPW wave to the spoof SPPs. To realize adiabatic transition, a flaring ground is designed to overcome the impedance mismatch between CPW and the spoof SPPs structure. The curve of the flaring ground is governed by

$$
\begin{aligned}
& \mathrm{y}=\mathrm{C}_{1} \mathrm{e}^{\mathrm{ax}}+\mathrm{C}_{2}, \quad \mathrm{C}_{1}=\frac{\mathrm{y}_{2}-\mathrm{y}_{1}}{\mathrm{e}^{\mathrm{ax}_{2}}-\mathrm{e}^{\mathrm{ax}_{1}}}, \\
& \mathrm{C}_{2}=\frac{\mathrm{y}_{2} \mathrm{e}^{\mathrm{ax}_{2}}-\mathrm{y}_{1} \mathrm{e}^{\mathrm{ax}_{1}}}{\mathrm{e}^{\mathrm{ax}_{2}}-\mathrm{e}^{\mathrm{ax}_{1}}}\left(x_{1}<x_{2}\right), \text { where } \alpha=0.2, \quad\left(\mathrm{x}_{1}, \mathrm{y}_{1}\right) \text { and }
\end{aligned}
$$

$\left(\mathrm{x}_{2}, \mathrm{y}_{2}\right)$ are the starting and ending points of the curve $[10,18]$. In the transition area, see Fig. 4, the length $\mathrm{b}$ of the drilled holes change adiabatically. The third part is the proposed Notch filter with $p=5 \mathrm{~mm}, \mathrm{a}=2 \mathrm{~mm}, \mathrm{~h}=4.8 \mathrm{~mm}$.

Fig. 5 shows the transmission profiles for one side transition part as a function of the step for the variation of the length for neighboring holes. As a comparison, the result of the length $b$ for the drilled holes unchanged (i.e. step $=0$ ) was given in Fig.5. The insertion loss with the length $\mathrm{b}$ unchanged was quite lager than the variable case. When the step of the gradient holes decreases, the insertion loss decreases at first then changes a little when it smaller than $0.6 \mathrm{~mm}$. We choose the hole length varies from $\mathrm{h} 1=0.6 \mathrm{~mm}$ to $\mathrm{h} 8=4.8 \mathrm{~mm}$ with a step of $0.6 \mathrm{~mm}$ here. The simulated results are shown in Fig.6. It can be seen the proposed filter has two sharp notch bands located at $5.36 \mathrm{GHz}$ and $9.32 \mathrm{GHz}$ with a $10 \mathrm{~dB}$ notched fractional bandwidth of $1.61 \%$ and $1.29 \%$ respectively, and its rejection more than $20 \mathrm{~dB}$. In the whole pass-band, the insertion loss of the proposed waveguide is better than $3 \mathrm{~dB}$.

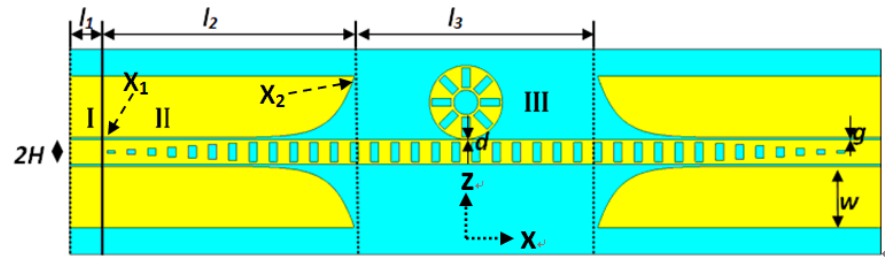

Fig.4. Schematic of the notch filter, in which $1_{1}=10 \mathrm{~mm}, 1_{2}=60 \mathrm{~mm}, 1_{3}=60 \mathrm{~mm}$, and $\mathrm{d}=0.1 \mathrm{~mm}$.

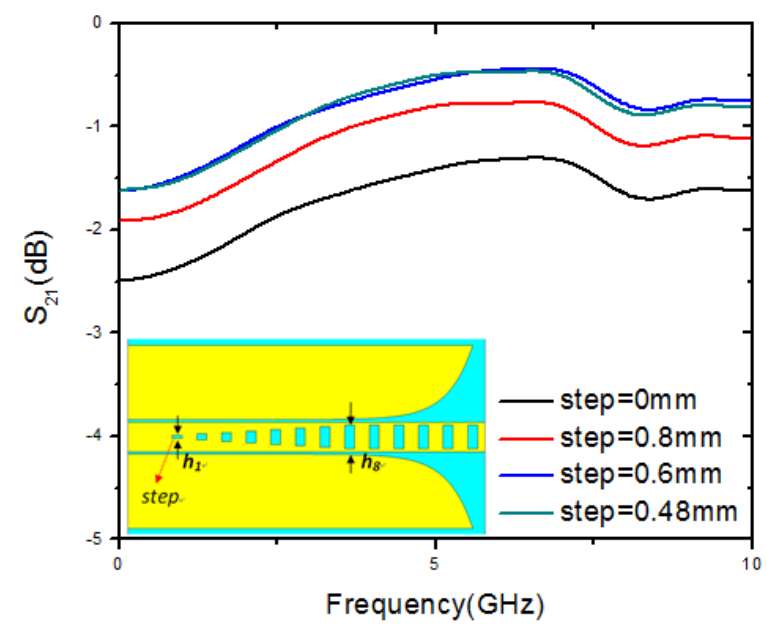

Fig.5. The influence of the gradient holes parameter on the insertion loss of the proposed structure. The insert shows the schematic of the structure.

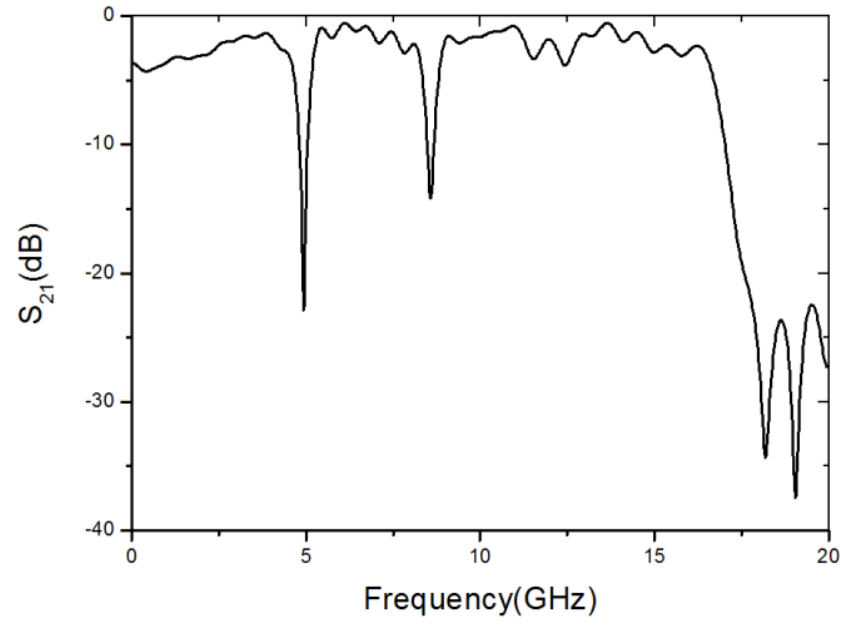

Fig.6.The simulated S-parameter of the UWB LPF with CPW in the microwave frequency.

\section{Conclusion}

We have proposed a notch filter for ultra-wideband microwave waveguide. A planar spoof SPPs ring resonator has been integrated into the ultra-wideband microwave waveguide based on ultrathin spoof SPP structures to provide wave propagation notch. The simulated results demonstrate that the SPP based ring resonators can be an alternative solution to existing techniques in creating a sharp band notch in UWB applications to suppress possible interferences with other communication systems. We have proposed an adiabatic transition relying on gradient hole-size and flaring ground, and the insertion loss is around $3 \mathrm{~dB}$ during the whole pass-band of waveguide, while having more than $20 \mathrm{~dB}$ rejections at $5.36 \mathrm{GHz}$ and $9.32 \mathrm{GHz}$ to avoid the existing WLAN and satellite communication signals. Compared with traditional notch filters, the proposed planar filter has subwavelength confinement, good adjustability and easy to integrate in the microwave integration circuits.

\section{ACKNOWLEDGMENTS}

This work was supported by 2016 Zhejiang Provincial Natural Science Foundation under Grant No.LY16F010010, in part by 2015 Zhejiang Province Public Welfare of International Cooperation Project under Grant No. 2015C34006.

\section{References}

FCC Report and Order. "In the matter of Revision of Part 15 of the Commission's Rules Regarding Ultra-Wideband Transmission Systems[R]", FCC 02-48, 2002.

Q. Li, Z. J. Li, C. H. Liang, and B. Wu, "UWB bandpass filter with notched band using DSRR," Electronics Letters, vol. 46, pp. 692-U46, 2010.

B. G. Xiao, P. Ye, Z. Y. Xie, and H. Liu, "Design of Ultra-Wideband Bandpass Filter Based on Composite Right/Left Handed Transmission Line", Advanced Materials Research, vol. 460, pp. 66-69, 2012.

J. Xu, W. Kang, C. Miao, and W. Wu, "Sharp rejection UWB bandpass filter with notched band," Electronics Letters, vol. 48, pp. 1005-1006, 2012.

X. L. Guo, C. Xu, G. A. Zhang, Z. J. Zhang, H. H. Yin, and Z. L. Wang, "Tunable low-pass MEMS filter using defected ground structures (DGS)," Solid-State Electronics, vol. 94, pp. 28-31, 2014. 
[6] B. G. Xiao, P. Ye, Z. Y. Xie, " A Novel Ring Slot Resonator UWB Bandpass Filter with Two Notch-bands," ADVANCED MATERIALS AND ITS APPLICATION, vol. 460, pp. 62-65, 2012.

[7] H. F. Ma, X. Shen, Q. Cheng, W. X. Jiang, and T. J. Cui, "Broadband and high-efficiency conversion from guided waves to spoof surface plasmon polaritons," Laser \& Photonics Reviews, vol. 8, pp. 146-151, 2014.

[8] X. Shen, T. J. Cui, D. Martin-Cano, and F. J. Garcia-Vidal, "Conformal surface plasmons propagating on ultrathin and flexible films," Proceedings of the National Academy of Sciences, vol. 110, pp. 40-45, 2012.

[9] L.-Y. Ou Yang, C.-H. Tsai, and S.-Y. Chen, "A Planar and Subwavelength Open Guided Wave Structure Based on Spoof Surface Plasmons," IEEE Photonics Journal, vol. 6, pp. 1-19, 2014.

[10] Z. Liao, J. Zhao, B. C. Pan, X. P. Shen, and T. J. Cui, "Broadband transition between microstrip line and conformal surface plasmon waveguide," Journal of Physics D: Applied Physics, vol. 47, pp. 315103, 2014.

[11] J. B. Pendry, L. Martin-Moreno, and F. J. Garcia-Vidal, "Mimicking surface plasmons with structured surfaces," Science, vol. 305, pp. 847-8, 2004.

[12] X. Liu, L. Zhu, Q. Wu, and Y. Feng, "Highly-confined and low-loss spoof surface plasmon polaritons structure with periodic loading of trapezoidal grooves," AIP Advances, vol. 5, pp. 077123, 2015.

[13] L. Liu, Z. Li, C. Gu, P. Ning, B. Xu, Z. Niu, et al., "Multi-channel composite spoof surface plasmon polaritons propagating along periodically corrugated metallic thin films," Journal of Applied Physics, vol. 116, p. 013501, 2014.

[14] B. C. Pan, Z. Liao, J. Zhao, and T. J. Cui, "Controlling rejections of spoof surface plasmon polaritons using metamaterial particles," Opt Express, vol. 22, pp. 13940-50, 2014.

[15] J. J. Wu, D. J. Hou, K. Liu, L. Shen, C. A. Tsai, C. J. Wu, et al., "Differential microstrip lines with reduced crosstalk and common mode effect based on spoof surface plasmon polaritons," Optics Express, vol. 22, pp. 26777-26787, 2014.

[16] A. Pors, E. Moreno, L. Martin-Moreno, J. B. Pendry, and F. J. Garcia-Vidal, "Localized Spoof Plasmons Arise while Texturing Closed Surfaces," Physical Review Letters, vol. 108, 2012.

[17] X. Shen and T. Jun Cui, "Planar plasmonic metamaterial on a thin film with nearly zero thickness," Applied Physics Letters, vol. 102, p. 211909, 2013.

[18] X. Shen and T. J. Cui, "Ultrathin plasmonic metamaterial for spoof localized surface plasmons," Laser \& Photonics Reviews, vol. 8, pp. 137-145, 2014

[19] F. J. Garcia-Vidal, L. Martín-Moreno, and J. B. Pendry, "Surfaces with holes in them: new plasmonic metamaterials," Journal of Optics A: Pure and Applied Optics, vol. 7, pp. S97-S101, 2005.

[20] A. P. Hibbins, B. R. Evans, and J. R. Sambles, "Experimental verification of designer surface plasmons," Science, vol. 308, pp. 670-2, 2005.

[21] H. F. Ma, X. P. Shen, Q. Cheng, W. X. Jiang, and T. J. Cui, "Broadband and high-efficiency conversion from guided waves to spoof surface plasmon polaritons," Laser \& Photonics Reviews, vol. 8, pp. 146-151, 2014.

[22] Y. Yang, H. Chen, S. Xiao, N. Asger Mortensen, and J. Zhang, "Ultrathin 90-degree sharp bends for spoof surface plasmon polaritons", Optics Express, vol. 23, pp. 19074-19081, Jul 152015.

[23] Bao Di Shen Xiao-Peng Cui Tie-Jun, "Progress of terahertz metamaterials", Acta Physics Sinica, vol. 64, pp. 228701, 2015.

[24] Tie Jun Cui and Xiaopeng Shen, "THz and microwave surface plasmon polaritons on ultrathin corrugated metallic strips", Terahertz Science and Technology, Vol. 6(2), pp. 147-164, 2013.

[25] Binggang Xiao, Sheng Kong, Jing Chen, and Mingyue Gu, "A microwave power divider based on spoof surface plasmon polaritons", Optical and Quantum Electronics, Vol. 48, pp. 1791-1796, 2016. 